\title{
ESTRATEGIA DE TRANSFORMACIÓN Y MODERNIZACIÓN DEL EJÉRCITO DE COLOMBIA
}

\author{
Crnl. RA Luis Eduardo Sánchez Aldana ${ }^{1}$
}

\begin{abstract}
Resumen
El Ejército Nacional siempre ha entendido la transformación como un proceso natural y necesario, y es por ello por lo que decidió llevar a cabo su proceso proactivo de cambio para adaptarse con la misma velocidad a la que evoluciona su entorno. De hecho, para el Ejército se constituyó en un cambio que abarcó todos los niveles de la Fuerza, mediante los cuales se vio obligado a adecuar la estructura organizacional, mejorar las capacidades operacionales y optimizar todos los procesos de soporte, ampliando la visión predictiva para enfrentar las amenazas inciertas del mañana. Para ello, como iniciativa del cambio planteado, se inició la construcción de una estrategia que permitiera alcanzar este objetivo, el cual daría respuesta a las necesidades futuras de la Fuerza; de manera tal que no sólo se establecieron criterios y guías institucionales para la construcción de la misma, sino también el diseño, desarrollo e implementación del plan Estratégico de Transformación Ejército del Futuro.
\end{abstract}

Palabras clave: Transformación, modernización, adaptación, capacidades, procesos, estructura, plan, cambio, optimización, innovación, transparencia.

\begin{abstract}
The National Army has always understood transformation as a natural and necessary process, and that is why it decided to carry out its proactive process of change, to adapt with the same speed at which its environment evolves. In fact, for the Army, the transformation is a profound change at all levels of the Force, which requires adapting the organizational structure, improving operational capabilities and optimizing all support processes, expanding the predictive vision, to face the uncertain threats of tomorrow. For this, the Force designed the transformation and modernization strategy that would respond to the future needs of the institution, establishing, not only the institutional criteria, but also, guidelines for the design, development, and implementation of the Transformation Strategic Plan for the Future Army.
\end{abstract}

Keywords: Transformation, modernization, adaptative, capabilities, processes, structure, plan, change, optimization, innovation, integrity.

\footnotetext{
${ }^{1}$ sanluis91@hotmail.com

Luis Eduardo Sanchez Aldana, coronel de la Reserva Activa del Ejército, es experto en transformación institucional y modernización organizacional. Profesional en ciencias militares, magister en Estrategia de Seguridad Nacional de la Universidad de Defensa Nacional (Washington DC), magister en administración de seguridad de la Universidad Webster (Missouri), Curso de Comando y Estado Mayor General del Colegio de Comando y Estado Mayor General (Kansas). De la misma manera, ha adelantado estudios de Transformación del Sector Seguridad (SSR) en la Academia Folke Bernadotte (Suecia), Transformación Internacional del Sector Defensa (IDT) en la Escuela Naval de Posgrados de EE. UU, y Liderazgo Estratégico en la Universidad de Oxford (Reino Unido); y representado al país en la Conferencia de Jefes de Transformación del Comando Aliado de Transformación de la organización del Tratado del Atlántico Norte (OTAN). Así mismo, ha asesorado en temas de trasformación a Fuerzas y Servicios de algunos países, entre los que se cuentan República Dominicana, Guatemala, Panamá, Ecuador, Perú, Brasil, Paraguay, Rwanda y Colombia.

INVESTIGADOR INDEPENDIENTE
} 


\section{Introducción}

$E^{1}$ Ejército siempre ha entendido la transformación como un proceso natural y necesario, es por ello por lo que decidió dirigir sus esfuerzos a estrategias que conduzcan al cambio (Comando de Transformación Ejército del Futuro, 2016), con el fin de adaptarse con la misma velocidad a la que evoluciona su entorno. Es así como durante este proceso se contemplaron tres consideraciones claves. La primera se refiere a un proceso de cambio y adaptación continuo; la segunda hace referencia a que la transformación y modernización se dan en un escenario de incertidumbre en el que debemos desempeñarnos de manera exitosa y ágil, y la tercera, que el desarrollo del proceso implica ser más eficientes y sostenibles, es decir, operar de acuerdo con la realidad económica del país y con las mejores prácticas identificadas en cuanto a seguridad y defensa (Ejército Nacional - Departamento de planeación, 2016).

Consecuentemente, surge la necesidad de articular todos estos aspectos para el desarrollo integral de la transformación y modernización de la Fuerza. Además del proceso, fue preciso el diseño y desarrollo de una estrategia que, además de consolidar las experiencias de la Fuerza, sirviera como guía y orientación para la construcción e implementación del Plan Estratégico de Transformación ${ }^{1}$ (PETEF), con miras a lograr los objetivos propuestos para la configuración del Ejército del Futuro.

\section{Diseño y desarrollo de la estrategia de transformación y modernización}

La estrategia de transformación institucional y modernización organizacional (Figura 1) nace desde el Direccionamiento Estratégico del Ejército Nacional, el cual es considerado la base de la gestión y proyección de la Fuerza.

En este sentido, el proceso de transformación permite responder a los retos que imponen el ambiente de seguridad y las expectativas de los colombianos y la comunidad internacional en el futuro, cimentándose en el conjunto de objetivos, políticas y estrategias que conducen al cumplimiento de la misión y la visión del Ejército, así como también en sus principios y valores institucionales (Ejército Nacional - Jefatura de Planeación y Transformación, 2015), pilares fundamentales de la evolución institucional.

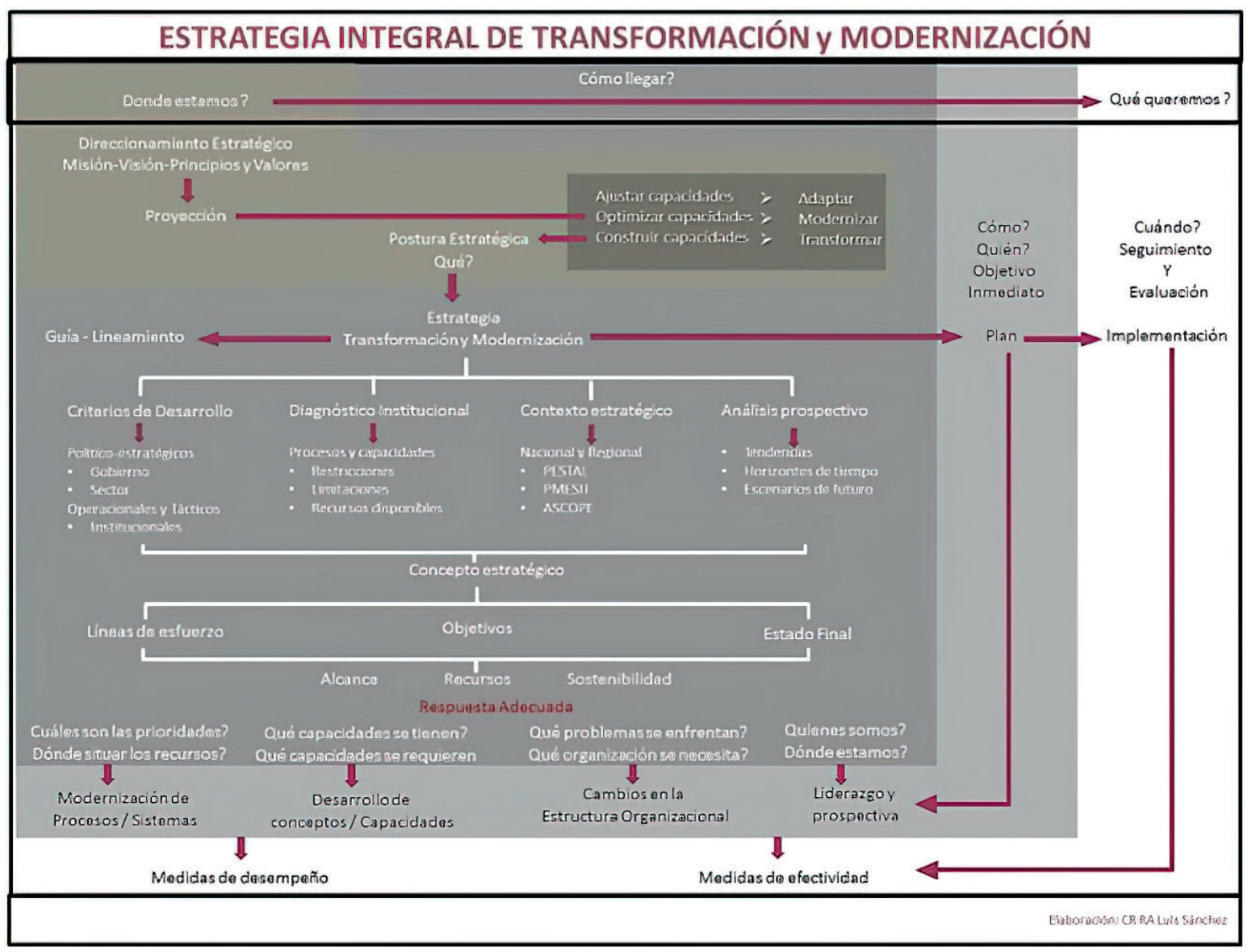

Figura 1. Estrategia integral de Transformación y Modernización de la Fuerza

Nota. Elaboración propia a partir de los conceptos del proceso de cambio y planeación estratégica del Ejército Nacional

${ }^{1}$ El Plan Estratégico de Transformación PETEF, tiene el propósito de establecer la hoja de ruta de la consolidación de una Fuerza preparada para afrontar los retos del futuro, en alineación con las políticas del Comando General de las Fuerzas Militares (COGFM), el Ministerio de Defensa Nacional (MDN) y el Gobierno Nacional (GN). 


\section{Postura estratégica}

Primordialmente se hizo necesario que la institución estableciera su postura con respecto al alcance del proceso. Es así como, basados en la teoría del Brigadier Jaime García Covarrubias": "Todo Ejército en evolución debe considerar tres aspectos para mejorar sus competencias y capacidades con el fin de cumplir con mayor eficacia, efectividad y eficiencia su misión" (refiriéndose a la Adaptación, Modernización y Transformación de las capacidades), se construyeron los conceptos dentro del proceso de transformación y modernización del Ejército. No obstante, los conceptos relativos a los cambios institucionales son de aceptación general, estos debían ser analizados con detalle al interior del Ejército dadas las características únicas y necesidades propias que diferencian un ejército de otro.

La adaptación está inmersa en el diario devenir de la Fuerza, haciéndose visible en la medida que se utilizan y se disponen los recursos con el fin de mejorar su desempeño, es decir, readecuar sobre la base de lo existente, siendo una responsabilidad inherente al mando en cualquier nivel; mientras que la transformación implica un cambio en la forma de empleo con nuevas capacidades en función de nuevas misiones, requiriendo el compromiso de la alta dirección para su ejecución; en tanto que, la modernización se refiere a la adecuación de la organización a nuevas necesidades, relacionándose un poco más con el rediseño institucional y la implementación de tecnología para aumentar la eficacia.

Por lo anterior, y teniendo en cuenta las diferencias conceptuales que se presentan entre la transformación y modernización, existen variables que las podrían asociar de manera simultánea o asincrónica; o ser dependientes, independientes o interdependientes. Sin embargo, todo gira alrededor de las necesidades y disponibilidad de recursos de la Fuerza.

De ahí se puede asegurar que la postura de la Fuerza en su momento hacía referencia al desarrollo simultáneo de los procesos de Transformación Institucional y Modernización Organizacional.

\section{Diseño de la estrategia}

Es preciso anotar que, además de tener una idea razonablemente clara de hacia dónde se llevaría la institución y de cómo articular el conjunto de iniciativas para lograrlo, la estrategia planteada por el Ejército estableció los criterios institucionales, identificó los problemas y necesidades, configuró el ambiente estratégico y visualizó los escenarios prospectivos para el desarrollo e implementación del proceso de transformación (Ejército Nacional, 2015), dando

\footnotetext{
${ }^{2}$ Brigadier Jaime García Covarrubias, oficial del Ejército de Chile y académico militar, experto asesor en transformación y modernización militar.

${ }^{3}$ Según el Ministerio de Defensa Nacional, el CRE-i es un comité conformado por expertos con el propósito de hacer una revisión a la estrategia y presentar soluciones innovadoras a un reto, problema o requerimiento de la institución.
}

así respuesta a las necesidades futuras de la Fuerza mediante el diseño de un plan que garantizara el cambio y la evolución de la organización.

\section{Criterios de desarrollo}

En el comienzo del proceso se recopilaron las guías institucionales de todos los escalones superiores, desde el gobierno nacional, pasando por el sector defensa, las Fuerzas Militares, hasta llegar a la propia Fuerza.

Dichos lineamientos orientarían el proceso en todos los niveles estratégicos, operacionales y tácticos del Ejército. Tales como:

a. La transformación mediante un proceso incluyente y participativo: no solamente los miembros de la institución, sino también la sociedad en general.

b. La transparencia institucional fortalecida en el cambio cultural: una transformación basada en principios y valores, que protejan la organización, los recursos y las personas.

c. El ajuste organizacional a la realidad presupuestal: proyectar los medios requeridos, de acuerdo con los criterios presupuestales del Ministerio de Defensa Nacional.

d. La alineación y articulación estratégica con el Gobierno Nacional, el MDN y el Comando General de las Fuerzas Militares (CGFM): un ejército parte de la fuerza conjunta, con mejores capacidades para el cumplimiento de los compromisos.

\section{Diagnóstico Institucional}

Posteriormente, y teniendo en cuenta que la transformación y modernización del Ejército son procesos de cambio continuos y dinámicos, se llevaron a cabo diagnósticos institucionales que permitieran establecer la situación actual y condiciones de la Fuerza para el desempeño de sus funciones, revisión de sus procesos y clasificación de sus capacidades operacionales para el cumplimiento de la misión.

Es así que los ejercicios académicos de análisis de la estructura organizacional empezaron en el año 2011 con la conformación del Comité de Revisión Estratégica e Innovación (CRE-i) ${ }^{3}$, seguido por el Comité Estratégico de Transformación e Innovación (CETI) ${ }^{4}$ en el 2012, y por el Comité de Diseño del Ejército del Futuro $(\mathrm{CEDEF})^{5}$ en el 2013. Sin embargo, cada uno de los ejercicios realizados tenía un objetivo propio y un nivel de desarrollo independiente.

\footnotetext{
${ }^{4}$ Mediante resolución 0167 de 2012, el comandante del Ejército conformó el Comité Estratégico de Transformación e Innovación (CETI), y delegó en él, la responsabilidad de construir el documento de diseño para la transformación del Ejército, con el objetivo de estar preparado para afrontar con eficiencia el futuro. ${ }^{5}$ Mediante resolución 0210 del 11 de febrero de 2013, el comandante del Ejército instaló el Comité Estratégico de Diseño del Ejército del futuro (CEDEF), con el objetivo de diseñar, planear, orientar, evaluar y dirigir la evolución del Ejército, y responder a las exigencias del sector defensa (áreas misionales).
} 


\section{DIAGNÓSTICO INSTITUCIONAL}

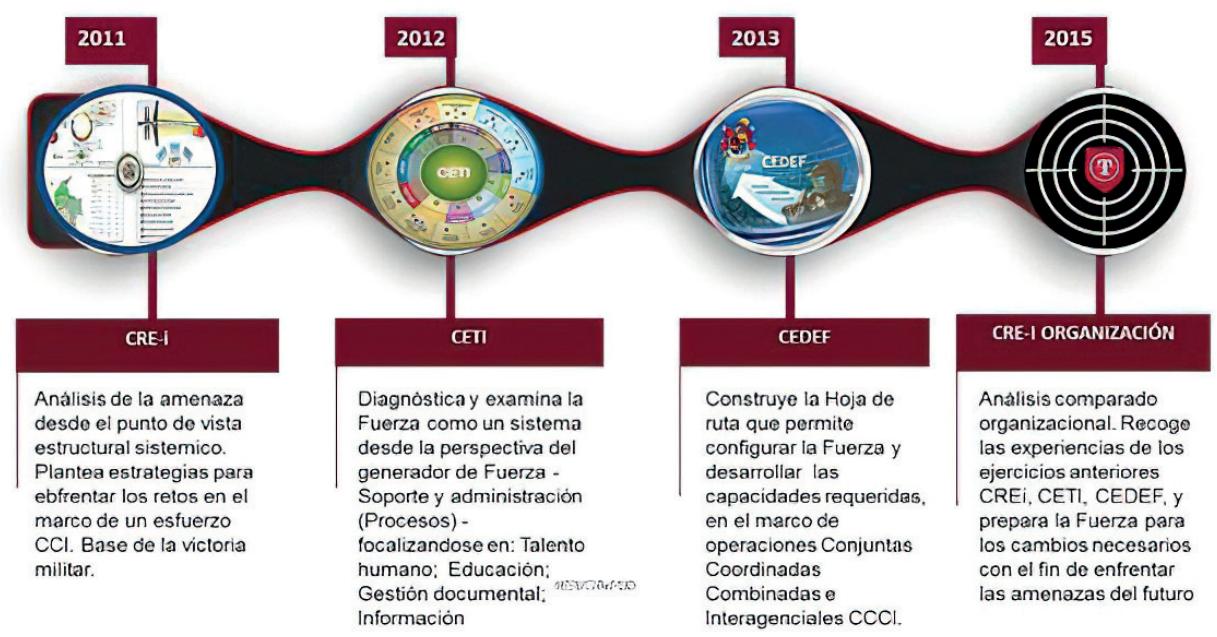

Figura 2. Diagnóstico Institucional

Nota. Elaboración propia a partir de los conceptos del proceso de cambio y planeación estratégica del Ejército Nacional

Es así como el CRE-i buscó aumentar la eficiencia y efectividad operacional a través del análisis del sistema rival o sistema de amenaza persistente (SAP) ${ }^{6}$. De forma similar, el CETI logró determinar las debilidades de la estructura de soporte, estableciendo a través de una serie de concentración de funciones los subsistemas que conformaban la Fuerza ${ }^{7}$. Así mismo, el CEDEF consolidó y clasificó las capacidades operacionales de la Fuerza, diseñando una metodología para su desarrollo ${ }^{8}$, fundamentados en el planeamiento basado en capacidades (Ejército Nacional - Jefatura de Planeación y Transformación, 2014).

Como resultado del desarrollo de estos ejercicios estratégicos de evaluación institucional, tanto de los procesos administrativos y de soporte, como de las capacidades operacionales, el Ejército consideró ciertas áreas y situaciones que, por su relevancia, se debían revisar profundamente, analizando los cambios que se pudieran generar, dentro de ella se destacan las siguientes:

a. El diseño de Fuerza: a través de una estructura organizacional altamente eficiente, más transparente y efectiva, fortaleciendo los canales de mando, supervisión y control.

\footnotetext{
${ }^{6}$ Para lograr enfrentar el sistema de amenaza persistente (SAP), se diseñaron planes operacionales con el propósito de reducir las amenazas, mediante la modernización de las capacidades operacionales bajo una estrategia Conjunta, Coordinada e Interagencial.

${ }^{7} \mathrm{Al}$ final del CETI, se presentaron propuestas en términos de proyectos e iniciativas tendientes a optimizar los procesos.

${ }^{8}$ Es así como, a través de la taxonomía de capacidades diseñada, se podría en primer lugar, desarrollar las

capacidades requeridas de la Fuerza para enfrentar las amenazas y, en segundo lugar, tener la base para la programación presupuestal acorde a las necesidades reales de la institución para cumplir su misión.
}

b. La configuración de Fuerza: mejorando su capacidad operacional a través de la racionalización de las unidades.

c. El empleo de la Fuerza: fortalecer las unidades tácticas, creando unidades más livianas con capacidad polivalente para cumplir las tareas impuestas.

d. La estabilización de la Fuerza: especialización del talento humano, buscando una mayor equidad en los procesos de selección, y una mayor inclusión de género.

e. La protección de la Fuerza: mediante el fortalecimiento del sistema jurídico integral de nuestros hombres.

f. La proyección de la Fuerza: sincronizando nuestra Fuerza con las otras fuerzas, agencias del Estado, otros países y fuerzas multinacionales.

\section{Contextualización estratégica}

Una vez realizado el diagnóstico institucional, el siguiente paso se relacionó con el análisis del contexto estratégico local, regional y global. Entre otros, se evaluaron factores determinantes como el proceso de paz, la pobreza, la desigualdad, el narcotráfico, la polarización política y la inestabilidad regional, que afectaban la realidad nacional, y por ende, también afectaban las condiciones que rodeaban el diseño y desarrollo de las operaciones militares. Sumado a esto, la dinámica de la violencia continuaba estrechamente relacionada con el negocio del narcotráfico y sus actividades conexas. 


\section{CONTEXTO ESTRATÉGICO}

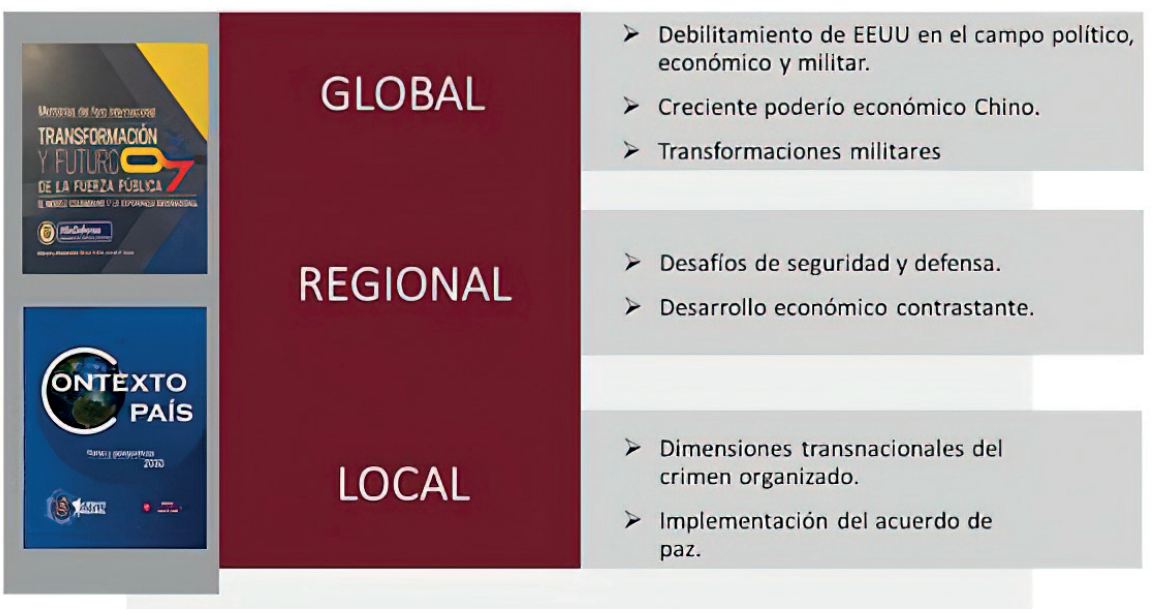

Figura 3. Contexto Estratégico

Nota. Elaboración propia a partir de los conceptos del proceso de cambio y planeación estratégica del Ejército Nacional.

En el orden global se tomaron en cuenta, además de las tendencias relacionadas a las potencias mundiales en los diferentes campos de gobierno, las transformaciones militares de los diferentes ejércitos del mundo. Es así como se efectuó un análisis comparado con los países que estaban llevando a cabo procesos de cambio en sus Fuerzas Armadas que sirvieran de referencia para proyectar el Ejército que requerían los colombianos (Ejército Nacional - Jefatura de Planeación y Transformación, 2015). En este orden de ideas, se revisaron los procesos de los ejércitos de Estados Unidos, España, Chile y Brasil. Adicionalmente, se estudió el Comando Aliado de Transformación de la Organización del Tratado del Atlántico Norte (OTAN), como motor y promotor de todos los cambios culturales, doctrinarios, de educación y de capacidades operacionales de la Alianza Europea.

Producto del análisis global se determinó que el mayor desafío en términos de seguridad y defensa se refería al carácter cambiante del conflicto. Un entorno dinámico con nuevos actores criminales, nuevas fuentes de financiación como la minería ilegal, nuevas amenazas, que, combinando lo asimétrico con las comunicaciones estratégicas que buscaban causar un mayor impacto en la población, recibieron un tinte de hibridez, más difícil de enfrentar.

Así mismo, en el entorno local, aunque el Gobierno Nacional avanzó en el proceso de paz con las Farc, las zonas con presencia de Grupos Armados Organizados seguían siendo complejas y con grandes dificultades, en principio por la influencia de las redes de economía ilícitas del crimen transnacional. Del mismo modo, los esfuerzos para explorar las posibilidades de diálogos de paz con el ELN no se materializaron, generando nuevos cambios en el contexto.
De manera similar, los Grupos de Delincuencia Organizada mantuvieron su poder intimidador, corruptor y generador de violencia gracias al auge del negocio de la producción y tráfico de drogas ilícitas. Por consiguiente, con el propósito de neutralizar la amenaza, fue conveniente entender estas organizaciones criminales como un sistema cuyo desempeño y evolución dependía del comportamiento de los subsistemas que lo componían. Como resultado, el Estado orientó su estrategia a través de un esfuerzo conjunto, coordinado e interagencial (Ministerio de Defensa Nacional, 2014), situación que obligó al Ejército a repensar la estrategia desarrollada hasta el momento, simultáneamente a la redefinición del diseño y reconfiguración de las capacidades para enfrentar las amenazas en el futuro inmediato y mediato.

\section{Análisis prospectivo y proyectivo}

En efecto, el ambiente operacional cambiante y complejo se analizó a la luz de las causas y consecuencias futuras, revisando las situaciones que se podían presentar con base en el estudio de los posibles escenarios del ambiente estratégico del mañana. 


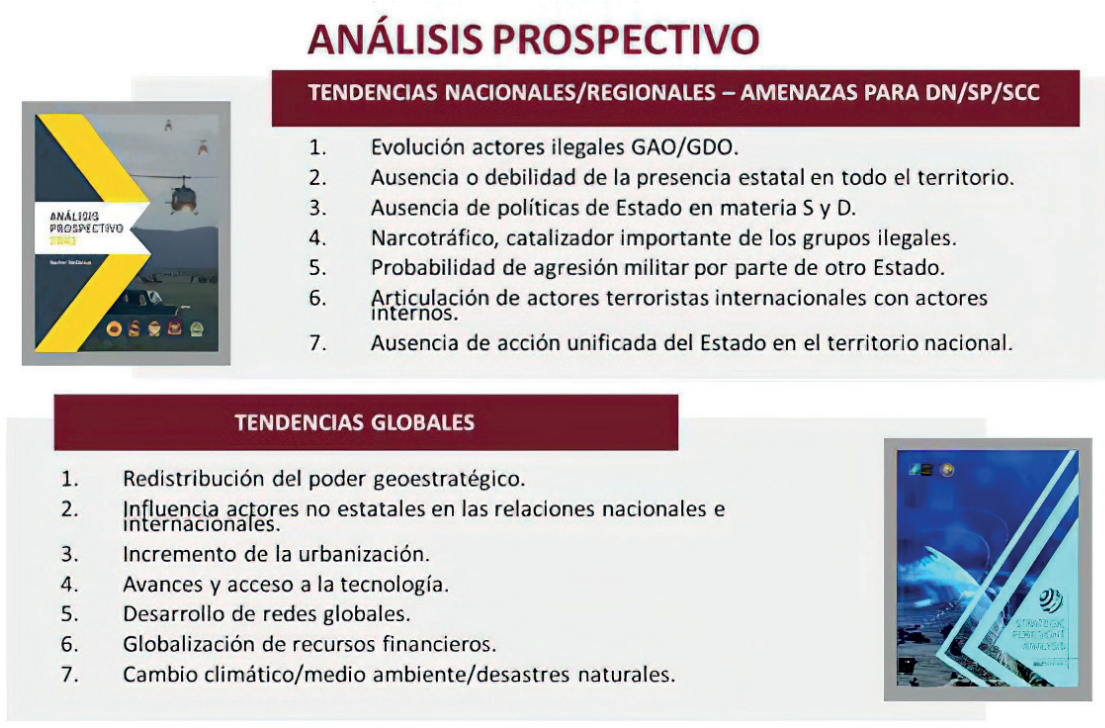

Figura 4. Análisis prospectivo

Nota. Elaboración propia a partir de los conceptos del proceso de cambio y planeación estratégica del Ejército Nacional.

De allí que, el análisis prospectivo realizado en el sector defensa (Ministerio de Defensa Nacional, 2015) permitió identificar algunos eventos que definirían el futuro contexto operacional, enmarcados en los siguientes puntos:

a. La evolución del conflicto como consecuencia de la atomización y articulación de los Grupos Armados Organizados al Margen de la Ley - GAOML con redes criminales internacionales, que desbordan las capacidades de la Policía: más amenazas de menor tamaño con mejores relaciones internacionales y mejor dotadas en tecnología.

b. Crisis o conflictos con amenazas externas: debido a intereses geoestratégicos o por la inestabilidad en Latinoamérica.

c. Evolución de la seguridad en el interior de los municipios: podría verse afectada por las condiciones socioeconómicas del país y la evolución de las amenazas actuales.

d. Presencia integral del Estado en el territorio nacional: la capacidad de las instituciones del Estado puede detener o acelerar la aparición de amenazas emergentes.

e. Narcotráfico y crímenes contra el medio ambiente: se consolidarán como las principales fuentes de financiación de las amenazas que atentan contra la estabilidad nacional.

Similarmente, el análisis de anticipación estratégico de la OTAN (NATO Allied Transformation Command, 2017) permitió visualizar el ambiente de seguridad del futuro, el cual se caracterizaría por su complejidad e incertidumbre. De hecho, la confluencia de varias tendencias políticas, sociales, tecnológicas, económicas y ambientales hacia el 2035 está redefiniendo el contexto de seguridad:

a. Campo político: se han dado mayores desafíos a la gobernabilidad, impulsados principalmente por la redistribución del poder económico y militar, y por la influencia de actores no estatales sobre los gobiernos nacionales y las instituciones internacionales.

b. Campo humano: las tendencias sociales con mayor influencia en el futuro son el cambio demográfico asimétrico, la rápida urbanización y sociedades cada vez más polarizadas. Estas predisposiciones, limitarán los presupuestos en seguridad y defensa, y aumentarán la conflictividad urbana y los disturbios sociales violentos.

c. Campo tecnológico: las tecnologías nuevas y emergentes ofrecen tanto oportunidades como desafíos. El avance tecnológico desafiará la interoperabilidad entre las naciones e instituciones, y a su vez, la delincuencia tendrá mayor oportunidad de explotar tecnologías fácilmente disponibles de una manera innovadora y potencialmente disruptiva.

d. Campo económico: aunque la globalización ha intensificado la integración económica, también ha alimentado la desigualdad social. Así mismo, un sistema financiero global cada vez más interconectado es más vulnerable a los ataques, y las redes financieras menos visibles y rastreables.

e. Campo ambiental: en este sentido, el cambio climático incidirá en el aumento de desastres naturales; de la misma manera, la demanda de recursos naturales, la seguridad alimentaria y el agua serán preocupaciones crecientes junto con pérdidas de biodiversidad.

Según el general de la Fuerza Aérea Francesa Denis Mercier, Comandante Supremo del Comando de Transformación de la OTAN:

El entorno de seguridad complejo y rápidamente cambiante seguirá siendo el principal impulsor de los esfuerzos de adaptación de la OTAN. Estos 
esfuerzos se centran en la transformación de la capacidad militar de la OTAN, para garantizar que la Alianza siga siendo relevante y creíble, ahora y en el futuro previsible, y pueda cumplir con sus tareas principales: defensa colectiva, seguridad cooperativa y gestión de crisis. Los hallazgos de este Informe (Análisis de Anticipación Estratégico de la OTAN) ayudan a la Alianza a comprender el presente y a visualizar el futuro, estableciendo un puente entre los dos, lo que permite que la OTAN se adapte y permanezca en forma para su propósito.

\section{Conceptualización estratégica}

Con este panorama, bajo las luces de los criterios institucionales, sumados al diagnóstico de proceso y capacidades, análisis del contexto y visión de futuro, se desarrolló el concepto estratégico de la Transformación y Modernización del Ejército que, por un lado, estableció el estado final del proceso, y por el otro lado, los objetivos para lograrlo.

\section{CONCEPTO ESTRATÉGICO}

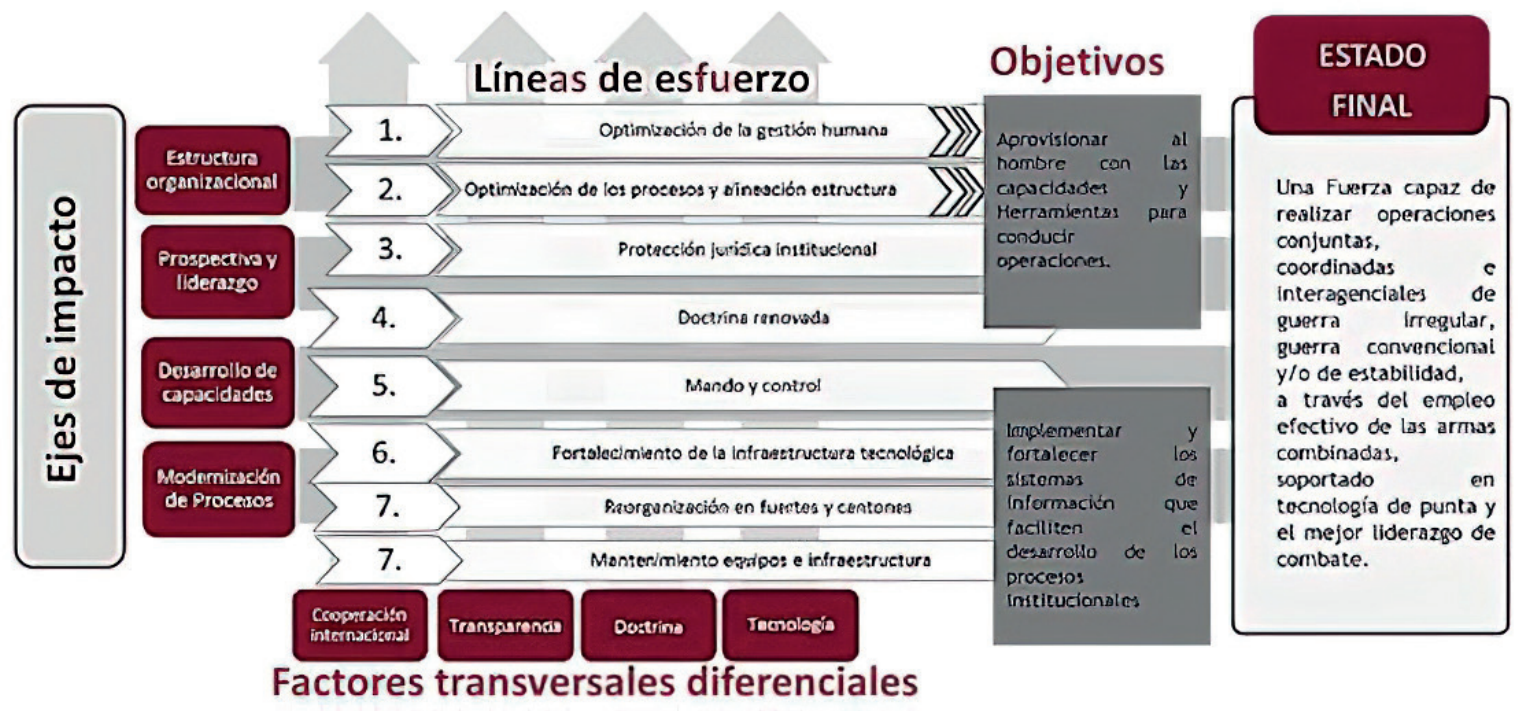

Figura 5. Concepto Estratégico de la Transformación

Fuente: Elaboración propia a partir de los conceptos del proceso de cambio y planeación estratégica del Ejército Nacional.

Básicamente, el estado deseado se refería a construir la Fuerza del futuro, capaz de interoperar con otras fuerzas y con los ejércitos más avanzados del mundo; un Ejército moderno basado en tecnología, referente regional y global.

Consecuentemente, el derrotero trazado para lograr este fin último se orientó, por un lado, a fortalecer al hombre y la mujer como el centro de la transformación, prepararlos, capacitarlos y dotarlos de las competencias necesarias para llevar a cabo el proceso de cambio al interior de la Fuerza; y por el otro lado, a mejorar los procesos de la organización, administrativos, de soporte y así mismo de los operacionales, creando una estructura fuerte, segura de sí misma y de lo que hace, y con las capacidades suficientes para cumplir a cabalidad con la misión constitucional.

De igual forma, se definieron dentro de la estrategia, además de la conceptualización, los objetivos específicos, medibles y alcanzables que contaran con recursos en un tiempo determinado. Es así como el proceso de transformación y modernización buscó garantizar el compromiso institucional del sector de defensa, haciendo un esfuerzo en la construcción de capacidades para la defensa de la soberanía y protección interna del territorio. Paralelamente, realizar tareas complementarias de contribución al desarrollo del país, protección del medio ambiente, atención de desastres, asistencia humanitaria, así como en la participación de misiones internacionales, promoviendo la relevancia institucional $\mathrm{y}$ facilitando la interoperabilidad $\mathrm{y}$ entendimiento común con otras Fuerzas (Ejército Nacional - Dirección de Planeación, 2013).

Adicionalmente, el proceso delineó, como objetivos específicos, diseñar estructuras organizacionales que optimizaran los procesos de soporte y fortalecieran la reorganización de la estructura de combate a través de la racionalización de las unidades, siendo más eficaces, efectivos y sostenibles de acuerdo con la realidad política, económica y social del país. Al mismo tiempo, se pretendía adoptar y adaptar las mejores prácticas identificadas en seguridad y defensa, que permitieran contar con un amplio rango de futuras alternativas y se llevaran a cabo las líneas de esfuerzo:

a. Optimización de la gestión humana. 
b. Optimización de los procesos y alineación de la estructura.

c. Protección jurídica integral.

d. Doctrina renovada y estandarizada.

e. Mando y control.

f. Fortalecimiento de la infraestructura tecnológica.

g. Mantenimiento de equipos e infraestructura.

En última instancia, el concepto estratégico permitirá definirá el alcance, los recursos y la sostenibilidad del proceso, generando respuestas que faciliten el diseño y desarrollo del plan estratégico de transformación. De esta forma, el concepto estratégico busca responder a las interrogantes ¿qué capacidades requiere la Fuerza para enfrentar las amenazas del futuro?, ¿cuál es la organización adecuada para cumplir los objetivos institucionales?, ¿qué procesos se deben optimizar para articular la organización?, y ¿cómo se deben prepara los líderes que construirán la visión de futuro y lo más importante que construirán la Fuerza del Futuro?

Podemos agregar que los objetivos de la transformación y modernización del Ejército de Colombia dieron origen a los ejes de impacto y a los factores transversales a todo el proceso con el fin de proyectar y robustecer la Fuerza para el 2030:

\section{Proceso autónomo, complejo y proactivo que permite a la
Institución evolucionar para anticiparse al entorno estratégjeo TRANSFORMACION N organizacional, el desarrollo de capocidades y la modernización de los procesos y procedimientos de la Fuerza}

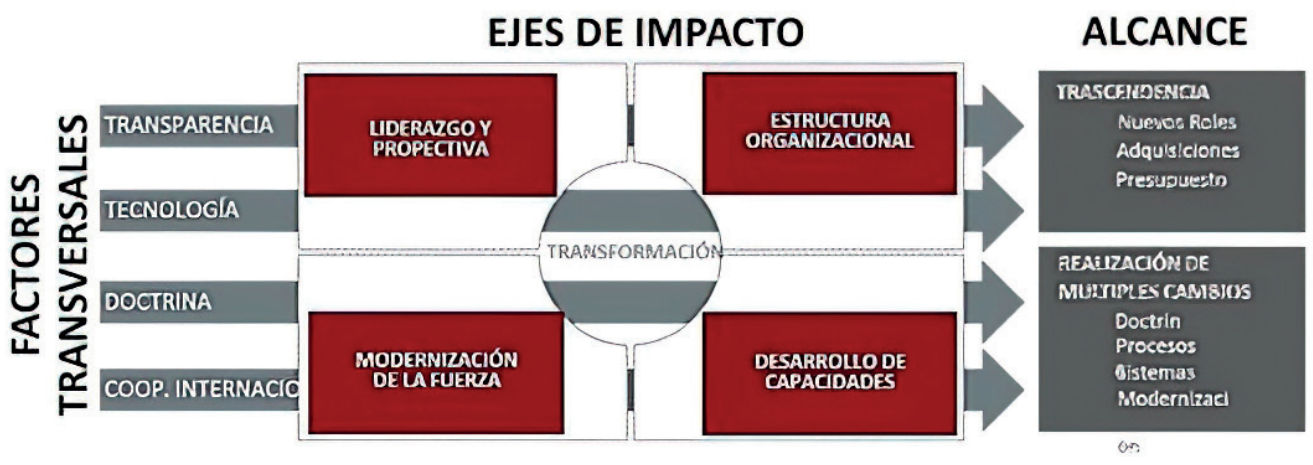

Figura 6. Factores transversales de la transformación

Nota. Elaboración propia a partir de los conceptos del proceso de cambio y planeación estratégica del Ejército Nacional.

\section{Ejes de impacto}

Son los pilares que soportan el proceso de transformación, adicionalmente articulan los objetivos y alinean las directrices, generando un efecto positivo sobre los sistemas componentes de la Fuerza. Dentro del proceso, se determinaron cuatro ejes:

a. La Estructura Organizacional: define los procesos, procedimientos, directrices y modelos de diseño de la Fuerza, para cumplir adecuadamente las metas propuestas.

b. Modernización de la Fuerza: se enfoca en la competitividad, implicando una innovación más que organizativa de infraestructura física y tecnológica, que permita más eficiencia, eficacia y efectividad.

c. Construcción de capacidades: mantiene las competencias necesarias para alcanzar sus propios objetivos, creando una Fuerza más profesional y capacitada.

d. Prospectiva y liderazgo: es en última instancia el líder quien visiona la naturaleza futurista del Ejército y requiere de la estrategia de largo plazo para tomar las riendas del futuro.

\section{Factores transversales}

Son elementos dinamizadores comunes a todo el proceso; en la práctica se dan de manera simultánea $\mathrm{y}$ tienen gran influencia sobre la transformación y modernización. Se consideran cuatro áreas esenciales que atraviesan la institución:

a. La doctrina: condiciona la organización, el material y el equipo, el personal, la infraestructura, el liderazgo, la educación y el mantenimiento.

b. La transparencia: genera cambios culturales enmarcados en la ética y en la implementación de principios de integridad, transparencia y rendición de cuentas.

c. Ciencia y tecnología: desarrollan iniciativas con el propósito de minimizar la dependencia tecnológica, y brindar sostenibilidad al Ejército.

d. La cooperación internacional: una Fuerza más preparada para interoperar en escenarios mundiales con los más altos estándares exigidos por las organizaciones internacionales.

\section{Plan Estratégico de Transformación}

Con el proceso en marcha, y con unos objetivos claros, medibles y alcanzables dentro del mismo, fue 
preciso diseñar el Plan Estratégico de Transformación Ejército del Futuro (PETEF) que permitiera aterrizar $\mathrm{y}$ hacer realidad la estrategia de transformación, modernización y cambio cultural, proyectando al Ejército hacia el corto, mediano y largo plazo.

Dicho plan se estructuró retomando los tiempos dinámicos de la transformación (Ejército NacionalComando de Transformación, 2016): iniciando con el T1.0 que culminaba en el 2018, el T2.0 en el 2022 y el T3.0 con horizonte en el 2030, priorizando los esfuerzos de la institución dentro de los tres tiempos. Así se diseñaría una transformación ordenada, optimizada y pertinente frente al ambiente operacional (Ejército Nacional, 2016). Dentro de este proceso de diseño se identificaron aspectos que superaban la perspectiva operacional y que requerían un análisis más profundo e integral que abarcara toda la institución y permitiera su proyección a un horizonte de tiempo mayor: hasta el año 2042 (Ejército Nacional - Comando de Transformación, 2016).

\section{TIEMPOS DINÁMICOS DE LA TRANSFORMACIÓN}
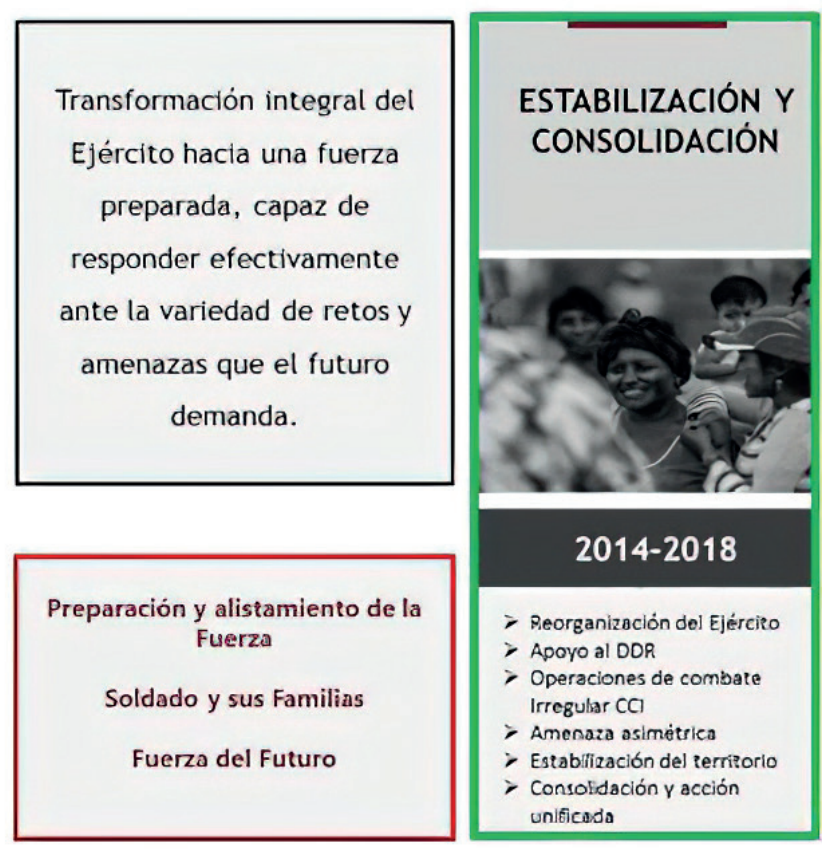

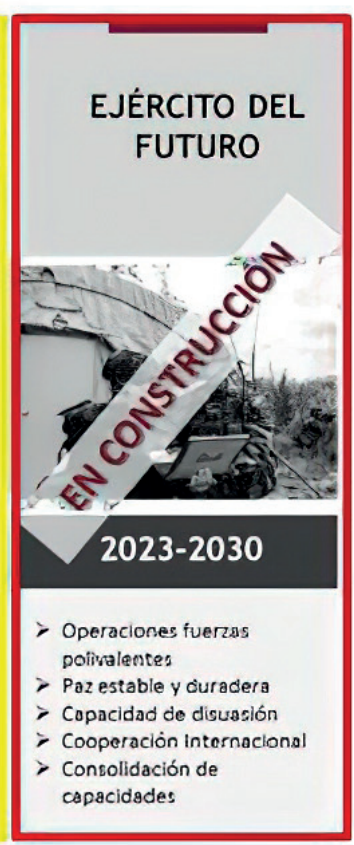

Figura 7. Tiempos dinámicos de la transformación.

Fuente: Elaboración propia a partir de los conceptos del proceso de cambio y planeación estratégica del Ejército Nacional.

Esencialmente el propósito del PETEF se orientó, por una parte, a fundamentar la toma de decisiones del nivel estratégico, integrando el planeamiento estratégico con la gestión presupuestal y de proyectos, enfocándose en el análisis de retos de interés para la institución y el diseño de planes de respuesta para enfrentarlos, a través de la priorización de proyectos estratégicos e integración de esfuerzos previos; y por otra parte, en la transformación integral del Ejército hacia una Fuerza capaz de responder efectivamente ante la variedad de retos y amenazas que el futuro demandará; esto se efectúa en cumplimiento de la misión constitucional dentro de las áreas misionales ${ }^{9}$ del Sector Defensa (Ministerio de Defensa Nacional, 2016).

En concordancia con el propósito, las líneas de esfuerzo del plan de transformación se focalizaron principalmente en potencializar las capacidades operacionales, fortalecer la sostenibilidad, la transparencia, entre otras.

\section{Implementación del plan de transformación}

Por último, no es fácil resumir los numerosos hechos palpables e imperceptibles que el Ejército Nacional llevó a cabo en el marco del proceso de transformación.

La transformación buscó desde el inicio mejorar y optimizar los procesos, potencializar su capacidad para neutralizar las amenazas y estar a la altura de los retos actuales y futuros, desarrollando un proceso continuo, rápido, firme y satisfactorio. Al término de la primera fase del plan estratégico de transformación en el año 2018 se pueden considerar algunos logros del proceso que se constituyeron en puntos de referencia en la ruta por recorrer, y benefician el objetivo de construir la Fuerza del Futuro.

\footnotetext{
${ }^{9}$ Las áreas misionales contienen responsabilidades tradicionales como la seguridad pública y defensa nacional, pero también presentan algunas oportunidades como la cooperación internacional, la contribución al desarrollo del país, la protección al medio ambiente y los recursos naturales.
} 


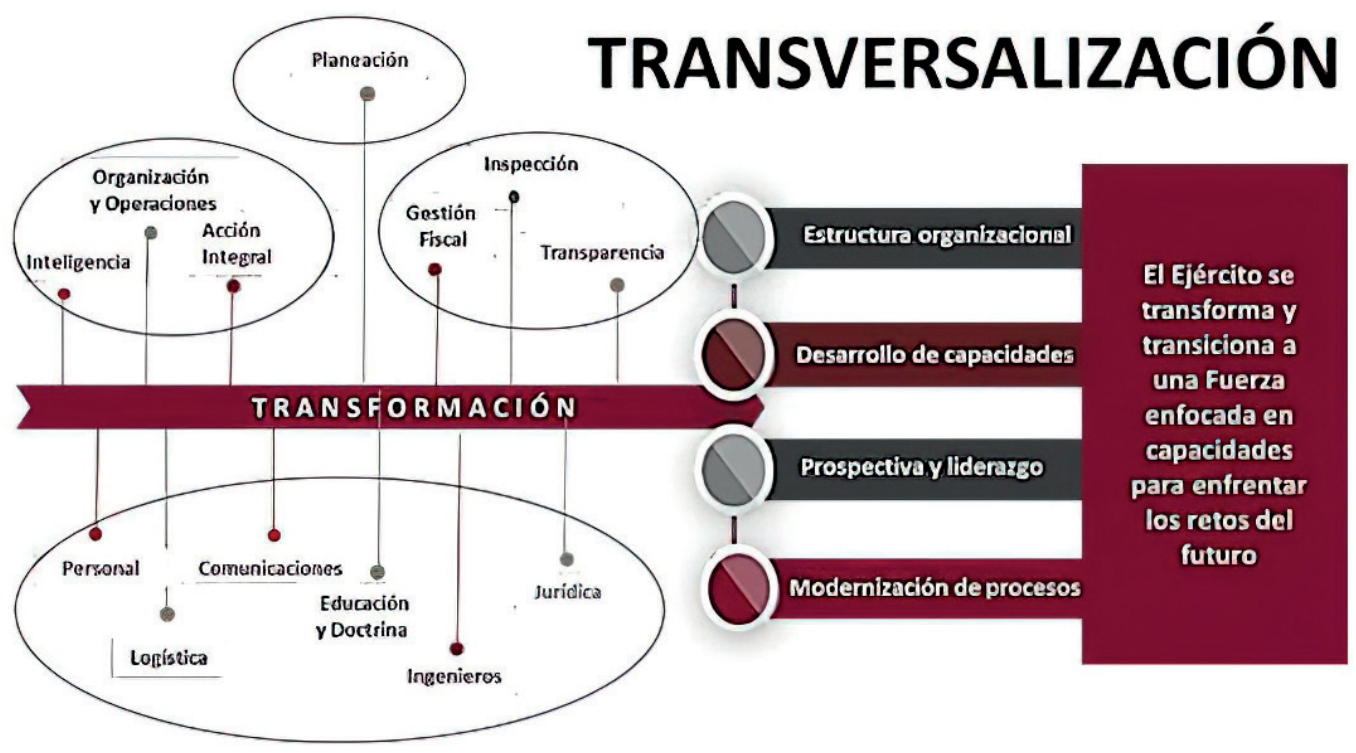

Figura 8. Ejes de impacto de la transformación.

Fuente: Elaboración propia a partir de los conceptos del proceso de cambio y planeación estratégica del Ejército Nacional.

Los siguientes son los avances más representativos ${ }^{10}$ de la evolución de la institución, y se presentan a través de los cuatro ejes de impacto del proceso de transformación, los cuales son transversales a toda la organización. En primer lugar, el ajuste a la estructura organizacional; en segundo lugar, la modernización de la Fuerza; en tercer lugar, la construcción de las capacidades, y por último, el liderazgo y la prospectiva (Ejército Nacional, 2018):

a. La Estructura Organizacional: definió los procesos, procedimientos, directrices y modelos de diseño de la Fuerza, para cumplir adecuadamente las metas propuestas.

- Reorganización de la estructura del Estado Mayor: mejoró la efectividad, la transparencia y la eficiencia a través de un proceso participativo.

- Administración del talento humano: se puso al servicio de la nación una Fuerza integra con perspectiva de género, fortaleciendo la cultura de autocuidado.

- Proyección Institucional: se diseñó el PETEF, generando proyectos para apalancar el proceso de transformación del Ejército Nacional.

- Fortalecimiento de la transparencia: se desarrollaron políticas y prácticas transparentes con el programa de Construcción de Integridad.

- Estandarización de la Doctrina: se da una apertura institucional, permitiendo acceder a la información relacionada con la doctrina terrestre.

\footnotetext{
${ }^{10}$ Avances del proceso de transformación presentados por el señor Mayor General Ricardo Gomez Nieto, comandante del Ejército Nacional, ante el cuerpo de Generales y Almirantes de las Fuerzas Militares. Conferencia realizada el 11 de abril de 2018 en el auditorio Domingo Rico de la Escuela Militar de Cadetes en Bogotá, DC.
}

b. Modernización de la Fuerza: se enfoca en la competitividad, implicando una innovación más que organizativa, de infraestructura física y tecnológica, que permita más eficiencia, eficacia y efectividad.

- Sistemas de control estratégico: para un control simultaneo relativo a la rendición de cuentas y a la transversalización de los sistemas de información.

- Investigación, Desarrollo e Innovación $(\mathrm{I}+\mathrm{D}+\mathrm{i})$ : optimizar los recursos y generar una mayor respuesta en seguridad y desarrollo tecnológico.

c. Construcción de capacidades: mantener las competencias necesarias para alcanzar sus propios objetivos, creando una Fuerza más profesional y capacitada.

- Creación de los comandos de apoyo: con el fin de apoyar a la comunidad, dar asistencia militar a las autoridades, y fortalecer la efectividad y el desempeño operacional de las unidades.

- Creación de unidades del orden estratégico: con el fin de contribuir al desarrollo del país, protección del medio ambiente, atención de desastres, y el logro de una mayor agilidad operacional para enfrentar las amenazas.

d. Prospectiva y liderazgo: Es el líder quien visiona la naturaleza futurista del Ejército y requiere de la estrategia de largo plazo para tomar las riendas del futuro.

- Internacionalización de experiencias y lecciones aprendidas: compartir experiencias y lecciones aprendidas, con Ejércitos de otros países.

- Programa de articulación institucional $F e$ en Colombia: mayor acercamiento a la comunidad, y mayor confiabilidad en la institucionalidad. 
- Academia: apertura el campus universitario más grande de Colombia, al integrar las instituciones bajo la sombrilla de las ciencias militares.

\section{Seguimiento y Evaluación}

Con el proceso de transformación y modernización de la Fuerza en marcha, y con el plan estratégico en ejecución, se estableció un mecanismo de verificación sistemática del cumplimiento de las metas establecidas determinando la eficacia, eficiencia y efectividad en la ejecución de los proyectos e iniciativas, para proponer argumentos a través del análisis de la toma medidas preventivas y correctivas que permitan alcanzar los objetivos institucionales.

De hecho, se realizaron dos ejercicios, el primero, de evaluación de la implementación de la nueva estructura del Estado Mayor, la cual marcó el inicio de la reorganización del Ejército y el segundo, de seguimiento a los proyectos e iniciativas del plan estratégico de transformación PETEF.

\section{Evaluación - Diagnóstico Organización del Ejército}

Mediante la realización de un ejercicio de diagnóstico (Ejército Nacional - Comando de Transformación, 2017), se pretendió mostrar el estado actual de la nueva estructura, su funcionamiento y lo más importante, que a partir de este análisis se fortaleciera la continuidad de la reorganización de la Fuerza (Componente Generador de Fuerza), y que facilitara la implementación de las unidades operacionales (componente Generador de Combate del Ejército).

Es bien sabido que la organización, como parte fundamental de la transformación, requiere la revisión y actualización permanente de los procesos adelantados para su desarrollo, con miras a fortalecer la proyección del Ejército del futuro; permitiendo, por un lado, acelerar la modernización, y por el otro lado, ajustar las funciones, los procesos y procedimientos, acoplándolos a los cambios estructurales.

Como resultado, se puede concluir en general que, la reestructuración del Estado Mayor es percibida como un cambio positivo para la institución, no obstante, se presentan algunas situaciones que deben ser revisadas y posiblemente ajustadas, como es de esperarse en este tipo de reformas, tales como la gestión del cambio, la caracterización de los procesos, y el estudio de perfiles para ocupar los cargos. La evaluación también permite evidenciar la persistencia de algún grado de desconocimiento por parte del personal de la naturaleza, implementación y ajuste de la nueva estructura ${ }^{11}$.

\footnotetext{
${ }^{11}$ El Comando de Transformación del Ejército COTEF, realizó el diagnóstico de la implementación de la nueva estructura organizacional de la Fuerza, que permitió establecer recomendaciones al mando institucional para mejorar el proceso
}

\section{Seguimiento - Plan Estratégico de Transformación PETEF}

El monitoreo del Plan Estratégico de Transformación PETEF, en su fase I, contó con un enfoque sistémico en donde se realizó, metodológica y metódicamente, seguimiento a los proyectos e iniciativas de construcción de futuro, que fundamentaron el desarrollo de propuestas en las perspectivas administrativa, logística y operacional, de esta manera se garantizó la estandarización, transparencia e identificación de oportunidades de mejora entre los procesos y la estructura organizacional.

\section{Conclusiones}

El proceso de Transformación Institucional y Modernización Organizacional del Ejército es considerado un acierto, mejorando la funcionalidad, el comando, la supervisión y el control al interior de la Fuerza. Sin embargo, el Plan de Transformación del Ejército del Futuro PETEF no garantiza por sí mismo el cumplimiento de la transformación institucional y de la modernización de la organización; es decir, no se constituye en un objetivo de la Fuerza, sino que se materializa como una herramienta que nos permite guiar y facilitar el entendimiento del impacto de las decisiones que hoy se toman sobre la seguridad y defensa del país.

Es así como la construcción de las fases subsiguientes del PETEF deben llevarse a cabo visualizando al Ejército que queremos en el año 2042. Esto permitirá realizar un ejercicio de planeamiento para identificar cómo debería estar configurado el Ejército en el año 2022 para cumplir la meta (Ejército Nacional - Comando de Transformación, 2016).

Este proceso de planeamiento con horizonte 2042 y con una "escala temporal" en el 2022 y en el 2030, deberá enfocar sus esfuerzos en la identificación y análisis de las posibles amenazas y, en consecuencia, deberá diseñar las capacidades requeridas para enfrentar estas situaciones.

Es allí donde la perspectiva del componente generador de combate del Ejército empezará a alimentar de requerimientos a la perspectiva del componente generador de Fuerza, impactando cada uno de los subsistemas del Ejército que deberán transformarse para desarrollar las capacidades de los tiempos futuros (Ejército Nacional - Comando de Transformación, 2016).

\section{Referencias}

Ejército Nacional - Comando de Transformación Ejército del Futuro. (2016). Transformación del Ejército de Colombia. Imprenta del Ejército.

Ejército Nacional - Comando de Transformación. (2016). Libro Estructural - Plan Estratégico de Transformación Ejército del Futuro. Imprenta del Ejército. 
Ejército Nacional - Comando de Transformación Ejército del Futuro. (2017). Diagnóstico Implementación Fase I Organización del Ejército. Imprenta del Ejército.

Ejército Nacional - Departamento de Planeación. (2018). Informe de Gestión 2018. Ejército Nacional.

Ejército Nacional - Departamento de Planeación. (2018). Informe ejecutivo de rendición de cuentas 2018. Ejército Nacional.

Ejército Nacional - Departamento de planeación. (2016). Plan Estratégico Institucional. Imprenta del Ejército.

Ejército Nacional. (2018). Logros del proceso de transformación del Ejército. Información del Comandante del Ejército (pp. 1-40). Ejército Nacional.

Ministerio de Defensa Nacional. (2015). Estudio de Análisis prospectivo. Ministerio de Defensa.

Ministerio de Defensa Nacional. (2017). Memorias al congreso 2016-2017. Ministerio de Defensa.

Ministerio de Defensa Nacional. (2016). Guía de Planeación Estratégica 2016-2018. Ministerio de Defensa. 